\title{
Von Florence Nightingale zu Adam Smith?* Wenn PatientInnen zu KundInnen und Gesundheitsdienstleistungen zu
Waren werden
}

\section{KARINA BECKER ${ }^{* *}$}

Das Spannungsverhältnis von Patientenwohl und wirtschaftlichen Zielstellungen im Gesundheitswesen ist schon länger Gegenstand von Debatten, die verschiedene Ebenen und (ExpertInnen-)Kreise durchziehen: Sei es als PatientIn, interessierte BürgerIn und BeitragszahlerIn, Sachverständiger oder Beschäftigter dieses Sektors - gesundheitspolitische Entwicklungen gehören aufgrund ihrer ethischen und sozialen Brisanz zu den vieldiskutierten Themen. Noch weitgehend unerforscht ist indes, welche konkreten Folgen die veränderten Rahmenbedingungen für die PatientInnenversorgung derzeit vor Ort haben. Der Artikel will einen Beitrag zur Schließung dieser Forschungslücke leisten. Auf der Basis umfangreichen qualitativen und quantitativen Materials, das in den letzten Jahren in der Pflegearbeit erhoben wurde, wird gezeigt, dass sich in diesem Bereich ein Wandel gesundheitsethischer Vorstellungen vollzieht, von dem auch die Berufspraxis der Pflegekräfte nicht unberührt bleibt.

Schlagwörter: Pflegearbeit, Emotionsarbeit, Gesundheitssystem, Ökonomisierung, Kommodifizierung

\section{Florence Nightingale to Adam Smith? When Patients Become Clients and Health Services Become Goods}

The tricky relationship between patient welfare and economic objectives in health care has long been a subject of debate. Whether they concern patients, interested citizens, contributors, health care experts or employees' health policy developments are highly controversial due to their ethical and social implications. However, the impact of the changed conditions in patient care is largely unexplored. The article aims to fill this research gap by presenting some empirical evidence. Based on extensive qualitative and quantitative material that has been collected in recent years in the nursing work this paper argues that there is an ongoing change in healthcare ethics, which has practical implications for care workers.

Keywords: Care Work, Emotional Work, Health System, Economization, Commodification

Beitrag eingereicht am 01.11.2012; nach doppelt verdecktem Gutachterverfahren überarbeitete Fassung angenommen am 16.08.2013.

** Dr. Karina Becker, Universität Trier, Universitätsring 15, D-54296 Trier, Tel.: +49-(0)6512012728, Fax: +49-(0)651-2013930, E-Mail: becker@uni-trier.de, Forschungsschwerpunkte: Finanzmarktkapitalismus, Gesundheit \& Markt, KMU, betriebliche Mitbestimmung. 


\section{Der Preis der Gesundheit}

Als Gut wird Gesundheit stets mit einem moralisch starken Impetus verhandelt, der bspw. an der weitgehend unumstrittenen Bestimmung von Gesundheit als Wert an sich erkennbar wird. ${ }^{1}$ Beim ethischen Bezug auf Gesundheit und deren Erhalt lässt sich allerdings im öffentlichen und wissenschaftlichen Diskurs eine Verschiebung der Argumentationsmuster und damit allgemeiner Maßstäbe konstatieren: Erfolgte die Auseinandersetzung um die Übertragung ökonomischer Logiken und Instrumente auf den Gesundheitsbereich lange mit Verweis auf die Wirtschaftlichkeits- und Rationalisierungsreserven der Krankenhäuser, die vollständig ausgeschöpft werden müssten, wird nunmehr auch die Begrenzung von medizinischen Versorgungsleistungen aus Kostengründen (im Sinne von Rationierung) als alternativlos erklärt. Im Kern drehen sich diese Debatten um die prinzipielle Frage, wie viel Gesundheit heute noch kosten darf bzw. soll, denn parallel zu diesen zunächst auf der diskursiven Ebene ablaufenden Aushandlungsprozessen vollzieht sich bereits eine Neuausrichtung des Gesundheitssystems in Richtung einer stärkeren Marktzentrierung. Für die Suggestion der Unausweichlichkeit einer Rationierung werden verschiedene Begründungszusammenhänge angeführt: Während zum einen darauf verwiesen wird, dass in der Praxis gegenwärtig ohnehin bereits Zuteilungsentscheidungen am Krankenbett getroffen werden müssen, für die ÄrztInnen und Pflegekräfte sich zukünftig auf explizite Regeln beziehen können sollen, werden zum anderen qualitätsbezogene Argumente herangezogen - zum Wohl bestimmter PatientInnengruppen zugunsten von Prävention, zum Vor- und Nachteil besonders kostspieliger Forschungsmedizin. Gemeinsames Moment dieser Positionen ist eine Sachzwangrhetorik, die eine Kostenreduktion als alternativlos und daher inzwischen auch unstrittig behauptet, ${ }^{2}$ ohne einen präzisen Beleg für eine andernfalls eintretende Prognose zu erbringen und häufig auch unter weitgehender Ausblendung konträrer Standpunkte (vgl. etwa Schöne-Seifert et al. 2006: 7). Entscheidend ist, dass diese Auseinandersetzungen zwischen Vertretern befürwortender und ablehnender Positionen einer (expliziten) Rationierung, einen Bargaining-Prozess charakterisieren, indem über gesundheitspolitische Weichenstellungen gerungen wird. Sie sind Teil des gesellschaftlichen Kampfs um die Definitionsmacht.

Die Grundüberlegung dieses Textes geht davon aus, dass die Ausweitung des Sachzwangarguments von der Rationalisierung auf die Rationierung medizinischer Leistungen von einer zunehmenden Kommodifizierung der Gesundheit angetrieben wird, die Teil einer sich seit Mitte der 1980er Jahre durchsetzenden neuen Hegemonie marktzentrierten Denkens ist. So war der Zeitraum nach dem Zweiten Weltkrieg durch einen gesellschaftlichen Konsens geprägt, der neben der materiellen Teilhabe an Pro-

1 Dies lässt sich beispielsweise an der Verfassung der Weltgesundheitsorganisation ablesen, die den Besitz des bestmöglichen Gesundheitszustandes als eines der Grundrechte jedes menschlichen Wesens, ohne Unterschied der „Rasse“, der Religion, der politischen Anschauung und der wirtschaftlichen oder sozialen Stellung definiert.

2 So stellte der Sachverständigenrat zur Begutachtung der Entwicklung im Gesundheitswesen für die konzertierte Aktion im Gesundheitswesen schon in seinem Jahresgutachten 1991 fest: „Eine Allokation von Ressourcen für einen Patienten auf Kosten eines anderen wird mit dem weiteren Fortschritt und dem Auftreten von Kapazitätsengpässen immer häufiger erfolgen müssen“ (Sachverständigenrat 1991: 208). 
duktivitätsgewinnen auch die Teilhabe aller Bürgerinnen und Bürger an medizinischtechnischen Fortschritten im Gesundheitsbereich einschloss. Die Frage, inwiefern medizinisches Urteilen und Handeln von Kostenerwägungen abhängig gemacht werden solle, war in dieser Phase eher nachgeordnet bzw. „blieb eher implizit“ (Kühn 2004: 25).

Im Gefolge der Krise dieses „fordistischen Entwicklungsmodells“ (Hirsch/Roth 1986) geriet auch dieser Konsens ins Wanken. ${ }^{3}$ Es findet seither - so die erste These dieses Beitrags - eine zunehmende Ausrichtung der medizinischen Versorgung an Marktprinzipien statt, die Ausdruck einer normativen Umorientierung ist oder auch erst zu dieser führt: Sie kennzeichnet den Einzug ökonomischer Logiken in gesellschaftliche Bereiche, die sich vormals in erster Linie an außerökonomischen Maximen orientierten. Darauf ausgerichtete Implementierungsmaßnahmen werden dann handlungsmächtig, wenn von gesetzgeberischer Seite der rechtliche Regelungsrahmen derart verändert wird, dass die Akteure im Gesundheitswesen ihre medizinischen und pflegerischen Entscheidungen, Therapien und Empfehlungen auch anhand ökonomischer Prämissen treffen - sie infolge dessen gegebenenfalls auch ummünzen.

Mit der Übertragung rentabilitätsorientierter Kalküle aus der Welt der Waren auf medizinische Sachverhalte und Probleme werden auch Gesundheitsdienstleistungen zunehmend zur Ware - deren Preis durch das Verhältnis von Angebot und Nachfrage bestimmt wird. Wenn, wie im Fall des regulierten Gesundheitsmarktes, ein pures, ZurWare-Werden' nicht oder nur unvollständig möglich ist, werden Wettbewerbe durch den Rückgriff auf Marktinstrumente künstlich simuliert oder inszeniert (vgl. Binswanger 2010). Damit vollzieht sich ein Paradigmenwechsel, in dem Gesundheit und deren Erhalt nicht mehr ihrem Selbstzweck genügen kann, sondern einen Zweck verfolgt, der darin besteht, ökonomische Zielgrößen zu (über-)treffen.

Kennzeichnend für die derzeitige Situation in der medizinischen Versorgung ist, dass die Normen und Ansprüche - der PatientInnen, aber auch der ÄrztInnen und PflegerInnen - an eine allgemeingültige Gesundheitsversorgung aus der fordistischen Phase und die neuen marktzentrierten Instrumente und Anreizsysteme in der Praxis aufeinanderstoßen. Die Ungleichzeitigkeit zwischen ,alten' Ansprüchen und ,neuen' Rahmenbedingungen - so die zweite These - treten in der Pflege besonders stark zu Tage. Dies liegt zum einen daran, dass die Beschäftigten dieses Sektors zwar ganz unmittelbar mit den neuen ,Marktzwängen' in Form des Kostendrucks (Ausdünnung von Personaldecken, zunehmende Arbeitsintensivierung etc.) konfrontiert werden, als Akteursgruppe jedoch über keine hervorgehobene gesellschaftliche Stellung verfügen; ihr gesellschaftliches Ansehen ist im Gegenteil in ihrer Selbstwahrnehmung eher ge-

3 Der Begriff Fordismus bezeichnet einen gesellschaftlichen Produktions- und Verteilungsmodus, der in Europa die Phase nach dem Zweiten Weltkrieg (in den USA bereits in den 1920er und 1930er Jahren) kennzeichnet. Er geht auf Henry Ford und die von ihm breit eingeführte Fließbandproduktion zurück, die der industriellen Fertigung zu enormen Produktivitätssprünge und daran anknüpfender Massenproduktion verhalf. Indem Ford diese Entwicklungen auch mit nachfragetheoretischen Überlegungen verband, schuf er die Voraussetzungen für einen von breiten Gesellschaftsschichten getragenen Massenkonsum und den Ausbau sozialer Sicherungssysteme. 
ring. ${ }^{4}$ Hinzu kommt, dass die PflegerInnen über keine funktionierende kollektive Organisierung verfügen, die sie bei der Durchsetzung ihrer Interessen unterstützen könnte. Dies hat unter anderem zur Folge, dass sie das Gefühl haben, auf viel weniger Verhandlungsmacht zurückgreifen zu können als etwa ihre Arzt-KollegInnen, ${ }^{5}$ die als relativ handlungsmächtige Gruppe ihre Interessen in den letzten Jahren besser zur Geltung bringen konnten. ${ }^{6}$

Der betriebliche Alltag in Krankenhäusern und ambulanten Pflegeeinrichtungen zeigt, dass sich die neuen Arbeitsbedingungen in der Regel anders auswirken als mit der Implementierung marktzentrierter Konzepte und Instrumente intendiert wurde. Dies bezieht sich auch auf die normativen Ansprüche an die eigene Arbeit der PflegerInnen, für die primär die humane Versorgung der PatientInnen leitend war oder auch (noch) ist. Dieser Beitrag beginnt daher mit einer historischen Rückschau auf die fordistische Phase nach dem Zweiten Weltkrieg (Abschnitt 2). Der ,Blick zurück nach vorn' soll verdeutlichen, welchen Veränderungen die Gesundheitsversorgung im aktuellen Marktkapitalismus unterliegt. Im dritten Abschnitt wird auf Basis eigenen empirischen Materials dargelegt, welche auch berufsethischen Konsequenzen der Einzug neuer marktzentrierter Steuerungssysteme für die Pflegearbeit in der Praxis hat. Für diese Analyse ist es sinnvoll zu unterscheiden, ob die PflegerInnen an der Referenzfolie fordistischer Errungenschaften und berufsethischer Standards festhalten oder aber sich zunehmend an den neuen Vorgaben und Versprechen des Marktes orientieren.

Die empirische Basis der Untersuchung bilden fünf Fallstudien (zwei Universitätskliniken, ein Krankenhaus mit einer explizit weltanschaulichen Ausrichtung, ein Krankenhaus in kirchlicher Trägerschaft sowie ein städtisches Krankenhaus), in denen die Autorin im Zeitraum von 2009 bis 2012 umfangreiches quantitatives und qualitatives Datenmaterial erhoben hat. Dazu gehören sowohl standardisierte Beschäftigtenbefragungen als auch (insgesamt 32) Interviews mit Pflegekräften, Pflegedienstleitungen, Ärzten und Interessenvertretungen (Betriebs- und Personalräte). ${ }^{7}$ Hinzu kommen Interviews und Befragungen in der ambulanten Pflege, darunter eine Studie zu den neueren Entwicklungen im Arbeits- und Gesundheitsschutz in ambulanten Pflegeeinrichtungen Thüringens. ${ }^{8}$

4 Dies lässt sich aus Sicht der PflegerInnen vor allem an dem aus dem wirtschaftsliberalen Lager kommenden Vorstoß ablesen, die Fachkräftelücke in der (ambulanten) Pflege durch Erwerbslose oder auch Beschäftigte anderer Branchen zu füllen, die dafür umgeschult werden sollen.

5 Ärzte genießen neben Anwälten und Priestern seit jeher eine hohe gesellschaftliche Anerkennung, vgl. dazu Freidson et al. (1979). Innerhalb des Gesundheitswesens gilt der Arztberuf als Leitprofession, vgl. Schwartz/Angerer (2010).

6 Geradezu prototypisch dafür ist der vom Marburger Bund - ursprünglich eine Standesvertretung der Ärzte im öffentlichen Dienst - geführte Streik der Krankenhausärzte im Jahr 2006, bei dem die Ärzte für sich einen eigenständigen Tarif durchsetzten.

7 Aus Platzgründen muss die Dokumentation des empirischen Materials in dieser verkürzten Form erfolgen. Die verwendeten Interviewpassagen werden bei wörtlichen Zitaten mit einer Signatur kenntlich gemacht. Die vollständigen Interviewtranskripte liegen der Autorin dieses Beitrags vor.

8 Vgl. die ausführlichen Befunde der quantitativen und qualitativen Erhebung zu den Arbeitsbedingungen in der ambulanten Pflege in Becker/Engel (2011). 


\section{Von der demokratischen Partizipation zur Marktselektion}

\subsection{Egalisierung der medizinischen Versorgung im Fordismus}

Wie viele andere Bereiche, blieb auch das Gesundheitswesen nie von ökonomischen Erwägungen unberührt. Erst die Ausweitung wohlfahrtsstaatlicher Elemente während der fordistischen Phase des Kapitalismus führte zu einer durchgreifenden programmatischen Egalisierung ${ }^{9}$ in der medizinischen Versorgung. Diese wurde von technologischindustriellen Fortschritten beispielsweise in der medizinischen Apparatemedizin aber auch von wissenschaftlichen Weiterentwicklungen in der Chemie-und Pharmaziebranche getragen und profitierte ebenso wie die für den Fordismus stehende Automobilindustrie von den enormen Produktivitätssprüngen der Massenproduktion. Zu den prägenden Struktur- und Funktionsbestandteilen der fordistischen Vergesellschaftungsform gehörten eine weitreichende Bürokratisierung und Durchstaatlichung, die unter anderem auf die Gewährleistung sozialer Sicherungssysteme und den Ausbau der sozialen Infrastruktur für breite Bevölkerungsschichten (Krankenhäuser, Altenheime) zielten (vgl. Hirsch/Roth 1986). Die damit einhergehende ökonomische und sozialstrukturelle Homogenisierung der Gesellschaft folgte jedoch keineswegs einer naturwüchsigen Entwicklung, sondern konnte nur in langen politischen Auseinandersetzungen über die Gestaltung des Wohlfahrtstaates, die Verteilung des erwirtschafteten Mehrprodukts und die De-Kommodifizierung des Gesundheitswesens erzielt werden. Als Kompensation für entfremdende, häufig auch disqualifizierende (Industrie-)Arbeit bot der korporatistisch-fordistische Regulationsmodus eine tarifvertragliche und politische Regulierung von Löhnen und Sozialausgaben, die den Beschäftigten eine kontinuierliche Verbesserung der materiellen Lebensverhältnisse und die Teilhabe an einem expandierenden Massenkonsum bot. Gleichwohl wurde die Primärmacht (Markt- bzw. Arbeitsplatzmacht) der Beschäftigten, beispielsweise Forderungen nach Lohnsteigerungen und verbesserten Arbeitsbedingungen, in Form von Arbeitskämpfen Nachdruck zu verleihen, durch ihre Bindung an Kollektivverträge eingeschränkt. Träger dieses, die fordistische Phase kennzeichnenden, Arrangements waren im Wesentlichen die Gewerkschaften, Arbeitgeberverbände und der Staat, die in konzertierten Aktionen korporatistische Regulierungen im Bereich der Sozialpolitik aushandelten (vgl. Müller-Jentsch 1993). Diese Errungenschaften führten insgesamt zu einem für breite Gesellschaftsschichten gleichen Zugang zur medizinischen Versorgung einer Demokratisierung im emphatischen Sinne -, die wie gezeigt werden soll, mit den Implikationen marktzentrierter Prinzipien und Instrumente nunmehr zur Disposition gestellt wird. Dabei handelt es sich jedoch eher um eine schleichende Aufkündigung des ,demokratischen Versprechens': Mit den im Fordismus etablierten Normen und Standards an eine humane PatientInnenversorgung wird weder von den Beschäftigten des Gesundheitswesens noch von den PatientInnen selbst explizit gebrochen. In den offiziellen Debatten zu diesem Thema - wie eingangs am Beispiel des Rationalisierungs-Rationierungsdiskurs gezeigt - deutet sich jedoch bereits ein Umbruch an, der

$9 \quad$ Zwar gibt es mit der Privaten Krankenversicherung auch Ausstiegsszenarien, dies gilt zu der Zeit jedoch nur für einen kleinen Teil der BürgerInnen. 
im Modus einer Sachzwangrhetorik eine allmähliche Aufkündigung des ,demokratischen Versprechens' einleitet.

\subsection{Krankenhäuser zwischen PatientInnenwohl und Gewinnmaximierung im Marktkapitalismus}

Der im Rahmen des 112. Deutschen Bundesärztetags 2009 getroffene Beschluss angesichts einer aktuell zunehmenden Kommerzialisierung und Marktorientierung des Gesundheitswesens, das ärztlich-ethische Bewusstsein für den Hippokratischen Eid ${ }^{10}$ zu stärken und sich mit der Approbation auch aktiv zu den ethischen Grundsätzen des Arztberufes zu bekennen ${ }^{11}$, öffnet den Raum für verschiedene Interpretationen: Zunächst lässt er sich als Bestrebung lesen, Strukturen und Prozessabläufe im beruflichen Alltag der Ärzte normativ statt monetär zu steuern. Appelliert wird an eine medizinische Ethik, die gemäß dem hippokratischen Eid das Heil bzw. Wohl der Kranken zur obersten Maxime bei der Ausübung des ärztlichen Berufes erklärt. Diese Prinzipien stehen nach Auffassung des Ärztetages im Widerstreit zu den aktuellen marktzentrierten Steuerungssystem der Krankenhausfinanzierung, die sich zunehmend in den binneninstitutionellen Logiken der Krankenhäuser und deren Akteure niederschlagen (vgl. Bär 2011; Gerlinger/Mosebach 2009). Vor dem Hintergrund dieser Entwicklung scheint den VertreterInnen des Bundesärztetags geboten, sich öffentlich von ärztlichem Handeln, das sich an ökonomischen Anreizen und nicht primär am PatientInnenwohl und deren Bedürfnissen orientiert, zu distanzieren.

Während Hippokrates als, Vater der Medizin' im Allgemeinwissen relativ prominent verankert ist, erfolgt die Auseinandersetzung mit ethisch-normativen Maßstäben und Standards in der Pflege eher abseitig im Kontext einer vor allem von den Berufsverbänden angestrebten Professionalisierung und beruflichen Emanzipation dieses Bereichs. Zurück gehen diese Bestrebungen auf Florence Nightingale, die in der zweiten Hälfte des 19. Jahrhunderts mit der Gründung einer Krankenpflegeschule mit eigenen Ausbildungsstandards wesentlich dazu beitrug, dass sich die Krankenpflege zu einem gesellschaftlich geachteten und anerkannten Berufsweg entwickelte. Seit 1953 gibt es auch für den Pflegeberuf berufsethische Standards, die im Ethik-Kodex des International Council of Nurses (ICN-Kodex) formalisiert wurden und seitdem kontinuierlich weiterentwickelt werden. Wie schon der Hippokratische Eid schreibt auch der ICNKodex die grundlegende berufliche Verantwortung der Pflegenden gegenüber pflegebedürftigen Menschen fest. Analog zum oben angeführten Beschluss des Ärztetags hält auch der Kodex der Pflegekräfte zu einer ethisch fundierten Verantwortungsübernahme, „zur Überprüfung eigenen Verhaltens und Handelns wie auch zur Entscheidungsfindung" unter erschwerten Rahmenbedingungen an (vgl. Juchli 1994: 514). Wenngleich sich ÄrztInnen und PflegerInnen - so lässt sich schlussfolgern - mit ei-

10 Der Hippokratische Eid - so die Begründung im Beschlussprotokoll - habe stets normierende, rational und pragmatisch motivierte Leitlinien für die Medizinerausbildung, das Arzt-PatientVerhältnis, den ärztlichen Beruf und dessen Handlungsstrategie geboten.

11 Bislang steht dieses passive Gelöbnis lediglich in den Unterlagen, welche jeder Arzt/jede Ärztin zu Beginn der Mitgliedschaft in der Ärztekammer erhält (vgl. Bundesärztekammer 2012). 
nem in der Regel unspezifischen Kostendruck konfrontiert sehen, ${ }^{12}$ sollte die Entscheidung darüber, wie dieser in konkrete Handlungen übersetzt wird, von ihnen selbst getroffen werden. Es sind eben jene ethisch-normativen Prinzipien im Sinne des hippokratischen Eids und des ICN-Kodex, die demnach konstitutiv für das Selbstverständnis medizinischen Personals sein sollen.

Das von Adam Smith betonte individuelle Interesse, in dem er die Triebkraft für den „Wohlstand der Nationen“ sah, mag zwar für viele Professionen zutreffend sein:

„It is not from the benevolence of the butcher, the brewer, or the baker that we expect our dinner, but from their regard to their own interest. We address ourselves, not to their humanity but to their self-love, and never talk to them of our own necessities but of their advantages. Nobody but a beggar chooses to depend chiefly upon the benevolence of his fellow-citizens." (Smith 1782/83: Wealth of Nations, Book I, Chapter II)

Insbesondere bei der Ausübung von personenbezogenen Humandienstleistungen soll das Streben nach Eigennutz jedoch von der natürlichen Suche nach Sympathie gebremst werden, wie es Smith selbst noch in seiner zuvor (1759) erschienen „Theorie der ethischen Gefühle“ begründet hatte: „Man mag den Menschen für noch so egoistisch halten, es gehören doch offensichtlich gewisse Prinzipien zu seiner Natur, die ihn am Schicksal anderer Anteil nehmen lassen und ihm deren Glück zum Bedürfnis machen, auch wenn er keinen anderen Vorteil daraus zieht als das Vergnügen, es zu sehen“" (Eckstein 1926: 1).

\subsection{Rationalisierung, Rationierung, Kommodifizierung}

Die Neu-Definition dessen, was als medizinisch notwendig bewertet wird, vollzieht sich vor dem Hintergrund einer Reihe gesetzgeberischer Änderungen, die dem Globalziel der Kostendämpfung folgend einen tiefgreifenden Wandel in der gesundheitspolitischen Ausrichtung induzierten. Festmachen lässt sich dieser Prozess an drei Bausteinen: Während Maßnahmen der Rationalisierung noch an die fordistische Logik anknüpfen, sind Rationierungsentscheidungen (als Verknappungsentscheidungen) bereits Ausdruck marktzentrierten Denkens und Handelns im Gesundheitswesen, die zu einer Re-Kommodifizierung medizinischer Leistungen führen. Diese Entscheidungen werden vor dem Hintergrund einer Neujustierung von Angebot und Nachfrage von Gesundheitsdienstleistungen getroffen.

Eingeleitet wurde dieser Wandel durch das Gesundheitsstrukturgesetz von 1992, mit dem das „Selbstkostendeckungsprinzip“ für Krankenhäuser durch verschiedene Formen prospektiver Finanzierung abgelöst wurde. Die Begrenzung der Steigerungsraten der Krankenhausbudgets (Budgetdeckelung), die zunächst nur bis 1995 als „Sofort-

12 Dieser ist auch darauf zurückzuführen, dass Parameter wie Effizienz und Effektivität im Krankenhaus insbesondere bezogen auf den Output nur schwer zu bestimmen sind. Auch der Sachverständigenrat zur Begutachtung der Entwicklung im Gesundheitswesen räumt in seinem aktuellen Sondergutachten „Wettbewerb an der Schnittstelle zwischen ambulanter und stationärer Gesundheitsversorgung“ ein, dass „,(..) angesichts ihres weitgefassten Bezuges und des vielfach komplexen Prozesses gesundheitlicher Leistungserstellung zumeist noch offen (bleibt) in welchen Bereichen und an welchen Stellen sich die betreffenden Effizienz- und Effektivitätsreserven befinden“" (Sachverständigenrat zur Begutachtung der Entwicklung im Gesundheitswesen 2012: 23). 
bremsung“" (BT-Drs. 12/3209: 41) fungieren sollte, später jedoch von der damaligen Bundesregierung aufrechterhalten wurde, verfolgt das Ziel, „die Krankenhäuser verstärkt zu wirtschaftlichem Verhalten (zu) motivieren“" (BT-Drs. 12/3608: 133) und insbesondere „die Verweildauer der Patienten zu verkürzen“ (BT-Drs. 13/381/94: 1). Wird die Budgetdeckelung von den einen auch deswegen als hoch problematisch bewertet, weil sie „,den Keim eines gesetzlichen Auftrags zur Rationierung medizinischer Leistungen in sich birgt" (Simon 2008: 6), sehen andere im Gegenteil darin einen Rationierungsmechanismus, mit dem die „Übernachfrage nach möglichst ausgedehnten Versicherungsverträgen (gemeint ist die Krankenversicherung) in ,vernünftige', das heißt finanzierbare Bahnen geleitet werden kann" (Kliemt 2006: 97). Statt die als unvermeidlich postulierte Leistungsbegrenzung in der Gesundheitsversorgung weiterhin zu verschieben, plädiert die zweite Position für eine Festlegung von Rationierungsinstrumenten, über die nicht auf der medizinischen, sondern auf der politischen Ebene entschieden werden müsse (vgl. Kliemt 2006: 104). Damit bleibt jedoch nicht nur offen, wo genau die Verantwortung für den Einsatz derartiger Leistungsbegrenzungen letztlich liegt, unklar ist auch, was mit der Befürwortung einer expliziten Rationierung insinuiert wird. Die wohl meist bemühte Forderung in der Kontroverse um Allokationsentscheidungen ist die nach Transparenz. ${ }^{13}$ Hierbei muss jedoch gefragt werden, ob eine nunmehr transparente Verteilung medizinischer Leistungen, als seien sie Waren, betroffenen PatientInnen tatsächlich hilft. Lässt sich Transparenz in diesem Kontext tatsächlich als Wert an sich begreifen, wie dies bei der Gesundheit der Fall ist? Überträgt man diese Diskussion auf die Mikroebene, muss zudem in Frage gestellt werden, ob aus der Explikation von Rationierungsbestimmungen folgt, dass die Akteure im Gesundheitswesen damit von ihrem ethischen Auftrag enthoben wären.

Zentral für die hier vertretene These einer schleichenden Aufkündigung des fordistischen Demokratieversprechens in der PatientInnenversorgung ist, dass sich mit der geforderten Verlagerung derartiger Entscheidungen vom Krankenbett auf die Makroebene ein Wandel andeutet. Hierbei erfahren traditionelle ethisch-normative Prinzipien und für einen bestimmten Zeitraum als selbstverständlich geltende Standards der medizinischen Versorgung, aber auch Identitäts- und Integrationsmechanismen der Organisation und ihrer Mitglieder einen Bedeutungswandel. Dies lässt sich an der vom Gesetzgeber forcierten stärkeren Marktanbindung des Gesundheitssystems nachzeichnen, für die Zusicherungen, wie ein Zugewinn an Transparenz und Freiheiten für die PatientInnen beispielweise bei der Krankenhauswahl vorgebracht werden. Um die

13 Andere Perspektiven setzen auch bei diesem Thema auf den Markt als „besten“ Koordinationsmechanismus und reklamieren dabei auch unter ethischen Gesichtspunkten zu argumentieren: „Of course, a free market allocates medical resources according to the price mechanism, which may seem to some to be unfair or arbitrary. Do not these aspects of the market violate dignity? While any allocation of scarce but necessary resources is going to have tragic aspects, there is no better way-ethically speaking - to allocate them than the market. The possibility of making private decisions regarding the benefits and costs of various treatment options, whether for minor illness or chronic disease, puts the choice in the patient's hands (as well as with her doctor and whomever else the patient wants to join the process, such as family or friends). In consultation with those close to her, the patient can assess the value of various treatments, considering the merits compared not only to their costs, and the benefits and costs of alternative options, but also other uses toward which those resources can be devoted, which are all subjective valuations“ (White 2010: 13). 
eklatante Informationsasymmetrie zwischen Anbieter und Nachfrager von Gesundheitsdienstleistungen zu schließen, werben Marktbefürworter wie der Patientenbeauftragte der Bundesregierung für Krankenhausrankings, die dem nunmehr autonomen Patientinnen bei der Suche nach „Deutschlands beste(n) Kliniken“ (Fokus 22/2012) helfen sollen. „Viele verlassen sich immer noch auf den eigenen Eindruck und vor allem auf Hinweise aus dem Bekanntenkreis“, so die Begründung. Leider stünde bei der Auswahl nicht die Qualität der Versorgung an erster Stelle, „Studien zeigen, dass Patienten die Zuwendung des Personals oder das Essen wichtiger ist", kritisiert der Patientenbeauftragte. Statt als PatientIn grundsätzlich auf eine optimale Krankenhausversorgung zu vertrauen oder auch darauf, dass der überweisende Arzt eine für den Einzelnen beste Entscheidung trifft, sollen PatientInnen demnach wie selbstbewusste Marktteilnehmer agieren und selbst nach dem „richtigen Krankenhaus“ suchen. An dieser Konzeption der PatientInnen als KundInnen lassen sich verschiedene Schwachstellen ausmachen.

(1) Zunächst ist ganz grundsätzlich danach zu fragen, ob man sich auch aus einer utilitaristischen Position heraus, tatsächlich darauf einlassen sollte, das für die medizinische Versorgung elementare System- und Personenvertrauen (in das Krankenhaus und das medizinische Personal) durch die Zusicherung von Transparenz zu ersetzen; nicht zuletzt deshalb weil für die PatientInnen damit neuartige Transaktionskosten (durch die Aneignung entsprechender Kompetenzen oder auch den Suchaufwand) entstehen würden. Krankenhäuser wiederum müssten - um besonders ,einträgliche' KundInnen (z.B. Privatversicherte oder PatientInnen, deren Diagnosen besonders gut abrechenbar sind, siehe unten) anzuwerben - ihre Ressourcen umlenken, indem sie in entsprechende Marketingstrategien investieren, wie dies von Privatklinikkonzernen bereits praktiziert wird.

(2) Von der versprochenen Wahlfreiheit des Anbieters von Gesundheitsdienstleistungen kann nur dann Gebrauch gemacht werden, wenn dem Nachfrager auch Zeit bleibt, an verlässliche - d.h. durch eine unabhängige Bewertung gewonnene - und für ihn relevante Informationen zu kommen. Der aktuelle Gesetzentwurf des Gesundheitsministeriums, wonach PatientInnen, die bei der Krankenhauswahl dem Rat ihrer Krankenkasse folgen, von zehn Euro Zuzahlung pro Tag befreit werden sollen, ist allerdings ein Beispiel für den Versuch diese Wahlfreiheit monetär ,abzukürzen'. So setzt der Rückgriff auf (Wahl-) Freiheitsversprechen immer auch entsprechende Ressourcen voraus, die in dem Fall den finanziellen Anreiz (zehn Euro pro Tag) ins Leere laufen ließen. Die behauptete Wahlfreiheit wird überdies dadurch konterkariert, dass sie auf dem Urteil eines in hohem Maße interessengeleiteten Akteurs basiert. Folgt man gar der Auffassung des derzeitigen Präsidenten der Bundesärztekammer „sind die Krankenkassen die einzigen Player im Gesundheitswesen, die an der Qualität überhaupt kein Interesse haben, sondern nur am Preis. Sie sind deshalb völlig ungeeignet, Patienten bei der Auswahl eines Krankenhauses zu beraten“" (o.V. 2012).

(3) Fasst man das Freiheitsargument des Marktes grundlegender, hieße das zudem, dass Freiheit auch impliziert, auf ein anderes Gut ausweichen zu können oder 
gänzlich darauf zu verzichten. Beide Optionen hat man als PatientIn in der Regel nicht. Weder verfügt dieser über die nötige Expertise ${ }^{14}$, Krankheiten und die Notwendigkeit darauf abgestellter Therapien einschätzen zu können, noch lässt sich anhand eines Krankenhausrankings die Güte des Behandlungssystems profund bewerten. Wenngleich im Wissenschaftsdiskurs immer wieder auf den Aspekt der körperliche Integrität von PatientInnen verwiesen wird, ist die Praxis doch vor allem davon geprägt, dass PatientInnen in erster Linie Schutzbefohlene sind, von denen - insbesondere in Notfällen - die angesprochenen Vorleistungen aus Zeitgründen ohnehin nicht erbracht werden können; die sich vielmehr darauf verlassen können müssen, die für sie beste medizinische Versorgung zu erhalten.

Sollten sich die angesprochenen Entwicklungen fortsetzen, könnten sich in absehbarer Zeit am Markt ,unabhängige Krankenhausberater etablieren, die sich etwa darauf spezialisieren, für ihre KundInnen das geeignete ärztliche und pflegerische Personal und das ihren Ansprüchen entsprechende Krankenhaus zu suchen und als Dienstleistung zu verkaufen.

Dass der Rekurs auf Wettbewerb und Markt zu neu entstehenden Abhängigkeiten bei den PatientInnen führt, zeigt auch das seit 2003 in Deutschland zur Anwendung kommende Abrechnungssystem nach diagnosebezogenen Fallgruppen (Diagnoses Related Groups - DRG). Danach rechnen Krankenhäuser mit den Krankenkassen nicht mehr nach der Liegedauer, sondern nach der Art der Diagnose ab, die nach einer bundesweit einheitlichen Pauschale vergütet wird. Obgleich bereits vor seiner Einführung vielfach auf die dem Abrechnungssystem innewohnenden problematischen Anreize hingewiesen wurde, verteidigte es der Gesetzgeber mit den gängigen Argumenten einer höheren Kostentransparenz und Effizienz, zu denen Krankenhäuser angehalten werden, wenn sie in einen Wettbewerb zueinander gesetzt werden. ${ }^{15}$ Zur ,bounded rationality' dieses Systems gehören: Als konkurrierende Unternehmen können Krankenhäuser dieser Logik folgend ihre Effizienz zum einen dadurch steigern, dass sie Mechanismen entwickeln mit denen es ihnen gelingt, unrentable PatientInnen', d.h. relativ schlecht bezahlte Diagnosegruppen, nur begrenzt oder gar nicht aufzunehmen oder auch umgekehrt medizinisch nicht notwendige Operationen und Therapien durchführen. ${ }^{16}$ Zum anderen halten mit diesem System auch selektive Kommodifizierungslogiken Einzug, die in Krankenhäusern analog zu privatwirtschaftlichen Unternehmen spezifische, abrechnungsfähige Vorgänge‘ zu Wertschöpfungsbereichen machen und andere, wie ein Teil der Pflegearbeit, hingegen zu ,angelagerten Bereichen und damit Kostentreibern werden.

14 Selektionsentscheidungen dieser Art sind in funktional ausdifferenzierten Gesellschaften nicht ohne Grund ExpertInnensache; dies entspricht der grundlegenden Logik gesellschaftlicher Subsysteme.

15 Mit der Einführung dieses Erlössystems wurde auch die Einführung und Dokumentation qualitätssichernder Verfahren gesetzlich festgelegt.

16 Das Spektrum impliziter Rationierungspraktiken ist breit und mittlerweile empirisch gut dokumentiert (vgl. etwa Bär 2011; Vogd 2006: 110ff.). 


\section{Pflegearbeit im Spannungsfeld von ,traditionellen' berufsethischen Normen und ,neuen' Marktanforderungen}

Was bedeutet nun die angedeutete Divergenz zwischen tradierten berufsethischen Orientierungen und Ansprüchen einerseits und den neuen marktzentrierten Vorgaben andererseits konkret für die Praxis der Pflegearbeit? Lange Zeit ging man davon aus, dass hier eine Rationalisierung der Arbeit wie in der industriellen Produktion oder auch der Verwaltung „,weder möglich ist noch angestrebt wird“ (Böhle 1999: 174). Auf arbeitswissenschaftliche Erkenntnisse gestützt, entwickelte sich stattdessen eine Praxis, die sich im Sinne einer ganzheitlichen Pflege der PatientInnenorientierung und Qualität verschrieb (vgl. Glaser 2006). Vollständige Pflegeaufgaben haben sich jedoch nicht nur für die PatientInnen, sondern auch für die Pflegenden selbst als vorteilhaft erwiesen (vgl. etwa Hacker 2005). Sie bieten einer Profession, bei der es darum geht, der Besonderheit der Situation und ihres Gegenübers bei der Arbeit Rechnung zu tragen, mehr tätigkeitsbezogene Ressourcen. Gleichwohl kamen bereits in den 1950er Jahren mit der Einführung der sogenannten Funktionspflege tayloristische Organisationsprinzipien zur Anwendung, von denen man sich eine höhere Effizienz versprach. Trotz einer in den 1970er Jahren aufflammenden Diskussion über Alternativen zur Funktionspflege wird diese als die seither die Praxis dominierende Form der Arbeitsorganisation bezeichnet (vgl. Rieder 1999). Mit den aktuellen Umbrüchen werden diese relativ frühen Rationalisierungsformen durch Angebotsverknappungen (Rationierung) und Inwertsetzungen von Gesundheitsleistungen (Kommodifizierung) ergänzt. Im Zuge dessen droht Pflegearbeit in der Tendenz zu einer um ihre Kernbestandteile gekappten Dienstleistung zu verkommen.

Dieser Wandel soll im Fokus des folgenden Abschnitts stehen. Dafür werden die derzeitigen Arbeitsbedingungen in der Pflege analysiert; Veränderungen, die ÄrztInnen betreffen, oder auch die Rückwirkungen auf die PatientInnen werden außen vor gelassen. Zunächst sollen die traditionellen Kernbestandteile der Pflegearbeit kurz umrissen werden, um im Anschluss daran darzulegen, durch welche strukturellen Veränderungen dieser Bereich geprägt ist.

\subsection{Traditionelle Pflege: Ganzheitlichkeit}

\subsubsection{Individualbetreuung}

Da sich Pflegearbeit vorwiegend auf Menschen richtet, deren Verhalten, Geschichte und Krankheitsbild nur sehr begrenzt standardisier- und kontrollierbar sind, sind Unwägbarkeiten und Unbestimmtheit geradezu Kennzeichen des Arbeitsalltags in der Pflege. Anders als bei Tätigkeiten an Maschinen sind PflegerInnen bei ihrer Arbeit mit der Eigenwilligkeit ihres ,Arbeitsgegenstands ${ }^{6}$ konfrontiert. ${ }^{17} \mathrm{Um}$ dabei der Individualität der PatientInnen Raum zu geben und zugleich die funktionalen Tätigkeiten ergebnisorientiert zu bewältigen, bedarf es des Einsatzes subjektiver Kompetenzen wie

17 Besonders prononciert gefasst hat dies Badura in seiner „Ko-Produktionsthese“. Der gemäß ist ein Spezifikum personenbezogener Dienstleistungsarbeit, dass die Qualität dieser in hohem Maße von der Interaktion zwischen Dienstleistungserbringer (Pflegekraft) und -nehmer (PatientIn) geprägt wird. Die PatientIn ist damit nicht einfach nur KonsumentIn, sondern Ko-AkteurIn. 
Intuition und Kreativität (vgl. Brucks 1999). Die Interaktion mit den PatientInnen wird dabei als Kernaufgabe von Pflegearbeit definiert.

\subsubsection{Organisation und Durchführung der Pflege}

$\mathrm{Zu}$ den traditionellen Aufgaben ganzheitlicher Pflege gehört zudem, dass betrieblichorganisatorische Aufgaben (dazu gehören klassische Tätigkeiten wie Wunden verbinden, Fieber messen, die Nach- und Vorbereitung der PatientenInnen für/vor einer Operation) so gestaltet werden, dass die PflegerInnen einen überschaubaren Zuständigkeitsbereich selbst verantworten und den individuellen Bedürfnissen der PatientInnen gemäß ausgestalten können. Dies ermöglicht ihnen einen vertrauensvollen, individualisierten Bezug zu den PatientInnen aufzubauen, deren Pflegeprobleme ,kennenzulernen' und mögliche -lösungen abzuleiten. Vollständige Aufgabenstrukturen bieten den PflegerInnen mehr Abwechslungsreichtum, Verantwortung und Lernerfordernisse und ziehen geringere Arbeitsbelastungen (durch Arbeitsunterbrechungen, zusätzlichen Informations- und Koordinationsaufwand etc.) nach sich (vgl. Glaser 2006a).

\subsubsection{Emotions- und Gefühlsarbeit}

Viele Pflegekräfte entscheiden sich bei ihrer Berufswahl bewusst für eine Arbeit, bei der sie auch selbst emotional stark involviert sind: Zum einen wird erwartet, dass sie die eigenen Emotionen dem Arbeitskontext entsprechend regulieren; Strauss et al. (1982) bezeichnen diese Arbeit an sich selbst als ,emotional work'. Zum anderen müssen sie in der Lage sein, die Gefühle der Patienten zu beeinflussen; für diese Arbeit an Anderen verwenden die Autoren den Begriff ,sentimental work'. ${ }^{18}$ Aussagen, wie die folgende sind geradezu typisch für die befragten PflegerInnen:

„Ich wusste schon ganz früh, eigentlich schon in der Schulzeit, dass ich Krankenpflegerin werden will. Ich war schon immer gern für andere Menschen da, habe immer mit ihnen mitgelitten.“ (05PK02_2011)

Solche Aussagen spiegeln die Bereitschaft, sich auch emotional - im Sinne von ,emotional' ${ }^{\varsigma}$ und ,sentimental work ${ }^{\varsigma}$ - auf andere Menschen einzulassen, wider.

\subsection{Pflegearbeit unter marktzentrierten Bedingungen: Patientenservice- management}

Wie erwähnt, geht es sowohl bei Formen der Rationalisierung als auch bei der Rationierung medizinischer Leistungen darum, Kosten zu minimieren - der Übergang zwischen beiden ist daher auch fließend. Darauf ausgelegte Maßnahmen und Entscheidungen lassen sich an drei zentralen Strategien festmachen.

\subsubsection{Verschlankung}

Die Tatsache, dass der größte Kostenfaktor in der Krankenversorgung die Arbeitszeit des ärztlichen und pflegerischen Personals ist - hierin demnach auch der stärkste He-

18 Zur Emotions- oder Gefühlsarbeit sind seit den 1990er Jahren eine Reihe von vor allem arbeitspsychologischen Studien und Konzeptionalisierungsansätzen erschienen (vgl. etwa Zapf 2002; Böhle 1999; Büssing/Glaser 1999). Auch die Arbeitssoziologie hat vielfach im Anschluss an Hochschild dieses Thema aufgegriffen (vgl. Badura 1990; Dunkel/Weihrich 2010). 
bel für Einsparungen besteht -, führt in der Praxis zu Verschiebungen in der ,Dienstleistungskette, die in Abbildung 1 schematisch dargestellt sind:

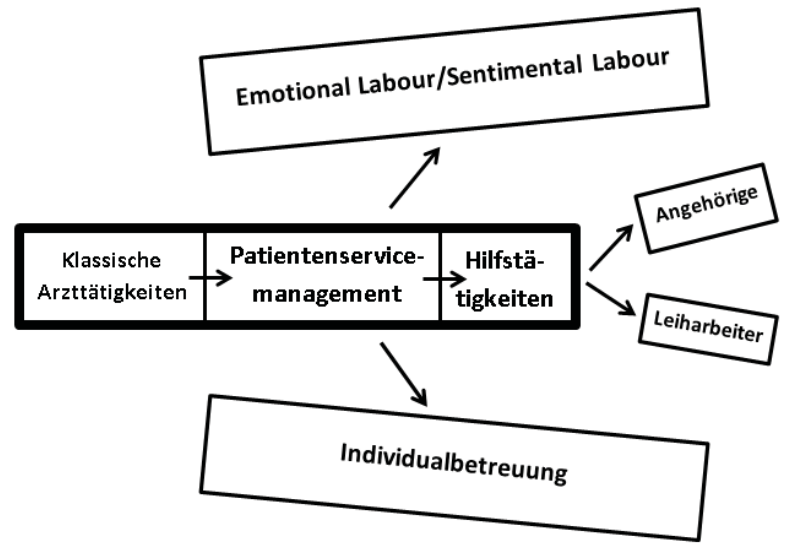

\section{Abbildung 1: Verlagerungsprozesse in der Pflegearbeit (Quelle: eigene Darstellung)}

Rationalisierungsstrategien in Form vertikaler Verlagerungen zielen in erster Linie darauf, die Fachkräftequote der Beschäftigten zu drücken. Im Zuge dessen delegieren hochqualifizierte ÄrztInnen vermehrt Funktionen an Pflegekräfte, ${ }^{19}$ die ihrerseits Tätigkeiten an Service- und Hilfspersonal abgeben. Für die Kostenreduktion in Form horizontaler Verschiebungen kreieren Krankenhäuser eigene Funktionen, wie die der operationstechnischen AssistentIn, die nicht staatlich anerkannt ist und daher auch keiner lohn-leistungsadäquaten Tarifierung unterliegt. Die vormals eher ganzheitliche Arbeit an den PatientInnen wird zunehmend fragmentiert, um die einzelnen Abläufe einer Wertschöpfungskette gleich - einer Kosten-Nutzen-Kalkulation zu unterziehen und so Effiziierungs- und Optimierungseffekte zu bergen.

Eines der untersuchten Krankenhäuser wurde in der Weise neu strukturiert, dass die ehemalige Organisation nach Bereichen, denen Fachkräfte mit Spezialisierungen für spezifische Krankheitsbilder zugeordnet waren, nunmehr durch eine interdisziplinäre Personalbesetzung abgelöst wurde. Ziel dieser Form der Kostenreduktion ist die Einsparung von Fachkräften, denn, interdisziplinäre Belegung heißt Abqualifizierung der Leute, die dort arbeiten“, so die befragte Betriebsrätin (07BR01_2012).

In allen hier untersuchten Krankenhäusern sind Pflegekräfte heute gehalten, ihr Handeln zunehmend an Prinzipien zeitökonomischer Rationalisierung und damit in erster Linie auf standardisierte und entsprechend kalkulierbare, abrechenbare Pflegeleistun-

19 Das Berufsrecht ist so angelegt, dass der Arzt die Verantwortung behält. Ein berufsrechtlicher Fortschritt wäre es dann, wenn damit eine faktische Übertragung einherginge (vgl. Jorzig et al. 2010). 
gen zu beschränken. Damit werden bestehende Berufe, wie die examinierte Pflegekraft sukzessive um ihre nicht-abrechenbaren Bestandteile - wie menschliche Zuwendungen aber auch Abweichungen von einem ausschließlich zweckrationalen Handeln - entkernt. Dies steht im markanten Gegensatz zur traditionellen Praxis, einer möglichst individuell gestalteten und ganzheitlichen Betreuung von PatientInnen.

Für einen Großteil der Pflegekräfte gehen marktzentrierte Steuerungsmechanismen mit einer deutlichen Verschlechterung ihrer Arbeitsbedingungen einher. ${ }^{20}$ Ein Beispiel dafür ist, dass die mit der Einführung des DRG-Systems intendierte Reduktion der Krankenhausverweildauer von PatientInnen auch für die Pflegekräfte eine massive Arbeitsverdichtung in Form von Zeit- und Leistungsdruck bedeutet. „Das Durchrauschen der Patienten“, so formuliert es die Pflegekraft eines Krankenhauses, das sich dem Prinzip ganzheitlicher Pflege verpflichtet fühlt, führe dazu, dass die Pflegekräfte ständig an der „Grenze der Arbeitsbelastungen sind, es flacht nie ab“ (07PK02_2012). Barg etwa die Vor- und Nachbereitung der PatientInnen auf bzw. nach einer Operation früher die Möglichkeit, diese auch etwas kennenzulernen und auch an deren Genesung teilzuhaben, gibt es die auch für das Krankenhauspersonal etwas ,entschleunigten Phasen“ heute faktisch nicht mehr: „Sobald die Patienten atmen und stehen können, sind sie weg, weil dann der Nächste kommt" (07PK02_2012). Um den Anforderungen einer hochverdichteten Organisation ${ }^{21}$ einerseits (bspw. die PatientInnen zur richtigen Zeit an die OP-Schleuse zu fahren, damit wiederum die Ärzte keine „Leerzeit" haben) und andererseits den Ansprüchen der PatientInnen, die keineswegs Abstriche in der Betreuungs(dienst)leistung machen wollen, , zu entsprechen, müssen PflegerInnen Strategien und Routinen entwickeln, die dafür sorgen, dass ihnen die Arbeit unter diesen zum Teil widersprüchlichen Rahmenbedingungen ,nicht völlig entgleist" (06PK02_2011).22

\subsubsection{Kommodifizierung}

Wie stark externe marktzentrierte Anforderungen im Zuge des Wandels der Krankenhäuser zu vornehmlich Dienstleistungsunternehmen die Frage beeinflussen, was Pflege eigentlich ausmacht, verdeutlicht die Beschreibung des Pflegedienstleiters eines städtischen Krankenhauses:

20 Einige Beschäftigte profitieren vom marktzentrierten DRG-System: Deren Logik führt auch dazu, dass das Krankenhausmanagement die OP-Laufzeiten ausdehnt und dafür den dort Beschäftigten außertariflich auch finanzielle Anreize setzt. Interessenvertreter verweisen darauf, dass diese Ausweitung auch die Arbeitszeiten der der OP vor- und nachgelagerten Bereiche verlängert; jene bleiben jedoch die Verlierer dieser selektiven Marktstrategie.

21 Diese Verdichtungsprozesse vollziehen sich vor dem Hintergrund einer Verschiebung von Marktgrenzen in die Organisation (vgl. Brinkmann 2011), die in der Regel den Abbau personaler und zeitlicher Puffer und/oder auch die Auslagerung ganzer Geschäftsbereiche (in einem Krankenhaus betraf das den Krankentransport, in einem anderen den gesamten Therapiebereich) nach sich zieht.

22 So haben die PflegerInnen in einem Krankenhaus bspw. in Eigeninitiative einen standardisierten Fragebogen entwickelt, in denen die Patienten Auskunft über ihre Eigenheiten (bspw. ob sie nach einem Eingriff viel Aufmerksamkeit und Kontakt wünschen oder ob sie lieber in Ruhe gelassen werden möchten etc.) geben können. 
„Es ist natürlich nicht mehr dieses Pflegen und nur dieses ,sich-um-Menschenkümmern', sondern es hat heute stärker mit Patientenservicemanagement zu tun. Also, der Patient als Kunde, der durch ein System gebracht werden muss, möglichst effizient und möglichst schnell. Die Pflegearbeit hat sich ganz stark zu einem Kundendienstleistungsverhältnis entwickelt.“"(10PL01_2012)

Pflegearbeit bedeute heute in erster Linie „Patientenservice-Steuerung“, so der Interviewpartner weiter.

Aus dem Grund sollte man sich seiner Auffassung nach auch heute nicht mehr ,aus humanitären Gründen für diesen Beruf entscheiden“ (10PL01_2012); lange Zeit am Bett einer PatientIn zu stehen, entspräche nicht mehr dem Arbeitsalltag in einem Krankenhaus, dafür müsse man schon in ein Hospiz wechseln. Auch der Personalrat eines Universitätskrankenhauses konstatiert nüchtern: „Keine Krankenkasse zahlt dafür, dass Patienten die Hand gehalten wird“" (05PR01_2010). Die Tatsache, dass Gefühlsarbeit warenförmig nicht abbildbar und damit auch nicht abrechenbar ist ${ }^{23}$ und die Auffassung vieler Pflegekräfte, dass diese aber geradezu konstitutiv für ihre Arbeit ist, konfrontiert diese mit einem kaum lösbaren Problem.

Im Zuge dieses Transformationsprozesses zu einem Patientenservicemanagement werden Elemente der traditionellen Praxis (Emotions-/Gefühlsarbeit sowie die Organisation der Pflege), wegrationalisiert‘. Die Funktionsfähigkeit der damit geradezu skelettierten Pflegearbeit beruht unter anderem auch darauf, dass Tätigkeiten vermehrt auf andere Akteure abgewälzt und damit externalisiert werden (vgl. Abschnitt 3.2.3).

Rationalisierte Arbeitsorganisationen und -bedingungen grenzen die Möglichkeiten für ,sentimental work' ein, wodurch eine Kluft zwischen den realen Möglichkeiten und den Ansprüchen der PatientInnen und Angehörigen, aber auch denen der Pflegekräfte selbst entsteht. Für die Pflegekraft einer Universitätsklinik haben sich die Arbeitsbedingungen nach Jahren kontinuierlichen Stellenabbaus derart verschlechtert, dass der „Anspruch an die eigene Arbeit immer mehr zu einem Minimalziel (schmilzt), das lautet: hoffentlich überleben alle in meiner Schicht“"(05PK03_2010).

Diese Diskrepanz führt bei den Beschäftigten zu Stress und Unzufriedenheit, die in vielen Fällen zu einer, ethischen Belastung'werden:

„Wenn der Dauerstress überhaupt nicht mehr aufhört, wenn sie keine qualifizierten Leute kriegen, wenn die eigenen Leute keine Luft haben, sich mal intensiv um die Patienten zu kümmern, dann vergisst man völlig, dass da jemand liegt, der eine Persönlichkeit ist, der Ansprüche hat. Es wird nur noch abgearbeitet. Das ist ganz furchtbar. Der Patient ist nur noch Objekt.“ (05PK04_2010)

Die Reduktion der PatientInnen auf einen Arbeitsgegenstand im medizinischpflegerischen Produktionsprozess zieht nach sich, dass ihrer Subjektivität keine Rechnung mehr getragen werden kann und die PatientInnen in der Tendenz zu einem Tä-

23 Die Nicht-Quantifizierbarkeit von Gefühlsarbeit ist auch ein starkes Argument gegen den Vorschlag des Sachverständigenrats 2007, der vorsah, das DRG-System für ein bestimmtes Spektrum von ausgewählten Krankenhausleistungen für den Preiswettbewerb zu öffnen. Danach hätten Krankenhäuser mit den Krankenkassen selektive Verträge mit speziellen Preis- und Qualitätsvereinbarungen abschließen können (vgl. Sachverständigenrat zur Begutachtung der Entwicklung im Gesundheitswesen 2007). 
tigkeitsobjekt werden. Aus der Forschung ist bekannt, dass Akteure in der Lage sein müssen, auch den eigenen ,Gefühlshaushalt' erfolgreich zu regulieren, um die Gefühlswelt anderer zu beeinflussen. Mit dem Auseinanderfallen eigener Ansprüche an die Profession und den von der Organisation vorgegebenen Bedingungen, wird demnach auch ,emotional work', also das Zeigen angepasster Gefühle, die der eigenen emotionalen Befindlichkeit nicht entsprechen, zu einer fremdbestimmten Arbeit (vgl. Hochschild 2003). Dass den PflegerInnen bei ihrer Arbeit ein komplexes Emotionsmanagement abverlangt wird, das unter ungünstigen Konstellationen gesundheitsbeeinträchtigend wirken kann, ist durch verschiedene empirische Studien, z.B. zum Zusammenhang emotionaler Dissonanz und Burnout in der Pflege belegt (vgl. Nerdinger/Röper 1999). Konnte der Rückgriff auf Ressourcen - wie die informelle Unterstützung durch die KollegInnen - den PflegerInnen früher dabei helfen, ihre spezifische Belastungssituation besser zu bewältigen, mutieren viele dieser Ressourcen unter den neuen marktzentrierten Rahmenbedingungen zu einer zusätzlichen Belastung (vgl. Becker et al. 2012). Dies hat vielfach gesundheitliche Konsequenzen, wie aus den Aussagen einer Pflegekraft hervorgeht:

„Man wird mit seinen Problemen hier völlig allein gelassen. Früher traf man sich wenigstens mal in der Teeküche und konnte sich auch mal über schwierige Patienten oder Sachen, die einem nahe gingen, austauschen. Das half schon. Heute bist du in der Schicht nur noch am Rennen und nimmst den ganzen emotionalen Ballast mit nach Hause. Und wenn du dann am nächsten Morgen wieder hier bist, hast du das Gefühl, du hast durchgearbeitet.“ (05PK05_2010)

\subsubsection{Externalisierung}

Die angedeutete Externalisierung von Pflegeaufgaben auf Organisationsexterne geschieht ebenfalls vor dem Hintergrund der Kostenreduktion. Dies ist dann beobachtbar, wenn die Personaleinsatzplanung so knapp bemessen wird, dass bestimmte Tätigkeiten (wie Waschen, Füttern, Anziehen etc.) vom angestammten und traditionell zuständigen Krankenhauspersonal nicht mehr oder nur noch ungenügend abgedeckt werden können, so dass diese zwangsläufig von den Angehörigen übernommen werden müssen ${ }^{24}$ - dies bezieht sich auch auf die Gefühlsregulierung der PatientInnen im Sinne von ,sentimental work'.

Eine andere Form der Externalisierung von Pflegearbeit stellt der Rückgriff auf Beschäftigte dar, die nicht zur Organisation gehören, dazu zählen Leiharbeiter oder auch selbstständige Pflegekräfte. Die momentane Situation in allen fünf untersuchten Krankenhäusern ist durch den kontinuierlichen Stellenabbau der letzten Jahre geprägt. Infolgedessen kann - auch die auf das Notwendigste beschränkte - Versorgung der PatientInnen nur noch durch zusätzliches Personal von außen gewährleistet werden (vgl. Bräutigam et al. 2010). ${ }^{25}$ Die Auswirkungen dieser Personalpolitik bekommen die

24 Die Strategie betriebliche Kosten und Funktionen an den Kunden auszulagern, ist schon länger Gegenstand vor allem arbeitssoziologischer Debatten. Sie wurde von Alvin Toffler (1980) eröffnet, der im „Prosumerism“ eine neue Form der Rationalisierung ausmachte. Die theoretische Weiterentwicklung des Phänomens des „arbeitenden Kunden“ geht vor allem auf Voß und Rieder (2005) zurück.

25 Die Leiharbeitskräfte werden als vollwertige Kraft je nach Bedarf in allen Stationen eingesetzt und müssen sich daher immer wieder neu mit den PatientInnen vertraut machen. Gerade auf den 
Stammbeschäftigten ganz unmittelbar zu spüren: „Wenn hier auf der Station Leasingkräfte eingesetzt sind, wird aus meinem 9-Stundentag ein 12-Stundentag“" (05PK06_2010). Dass der Rückgriff auf organisationsexterne Kräfte für die Stammbeschäftigten zu einer Intensivierung und Extensivierung der Arbeit führt, hat verschiedene Gründe: Zum einen wissen sie vielfach schlichtweg nicht, über welche Qualifikationen die LeiharbeiterInnen verfügen, ob sie für bestimmte medizinischen Apparate eingewiesen wurden und wer die Verantwortung dafür trägt, „wenn bei den Leiharbeitskräften etwas schief geht“ (05PK07_2010). Zum anderen sind die zusätzlichen Belastungen auch darauf zurückzuführen, dass die externen Pflegekräfte ausschließlich für die unmittelbare Versorgung der PatientInnen geleast werden und alle darüber hinausgehenden Tätigkeiten als informelle Zusatzaufgaben (Auffüllen von Behältern, Gespräche mit den Angehörigen etc.) allein von den Stammbeschäftigten eingebracht werden müssen. Der Einsatz von Leiharbeitern erfolgt damit vor allem auf dem Rücken der Stammbeschäftigten.

Insbesondere dann, wenn sich Leiharbeitskräfte neben materiellen Anreizen aus eben diesen Gründen gegen eine Festeinstellung entscheiden und damit den die Gruppe verbindenden Wert aufkündigen, gemeinsam zu einer bestmöglichen Versorgung der PatientInnen beizutragen, beginnt die interne Kollegialität und Solidarität unter den Beschäftigten zu erodieren. Eine Pflegerin sagte dazu im Interview:

„Früher war es überhaupt kein Thema mal für eine Kollegin kurzfristig den Wochenenddienst zu übernehmen, man konnte sich auch darauf verlassen, dass sie dies auch für mich tut. Heute hat man es hier ständig mit neuen Leuten zu tun und weiß ich, ob die das auch für mich machen würden? Das heißt dann eben auch, dass die Station auch mal unterbesetzt ist.“ (06PK02_2011)

Dieser der Marktlogik entsprechende Verlust etablierter binnenorganisationaler Integrationsmechanismen, der oft auch unter dem Stichwort ,Einzelkämpfermentalität ${ }^{\varsigma}$ diskutiert wird, konfrontiert auch das Management mit unintendierten Effekten, die die Effizienz der Organisation schmälern. Ein Beispiel dafür ist die Strategie, die Personalplanung von der Pflegedienst- oder Stationsleitung direkt auf die Beschäftigten zu verlagern, um so das Solidarprinzip zur Disziplinierung auszunützen - schließlich „fühlt man sich der Kollegin, die darum bittet, außerplanmäßig noch eine weitere Schicht zu übernehmen, eher verpflichtet“ (06PK02_2011). Auf diese Weise werden ,Notgemeinschaften' gebildet, die - wenn sie auf funktionierende Sozialbeziehungen gründen - strukturelle Defizite kompensieren können. Für das Management stellt sich daher die Aufgabe, auf die von den externen Beschäftigten in die Organisation getragene Handlungsorientierung, die auf eine Befreiung von sozialen Zwängen und Verantwortung zielen, mit entsprechenden Strategien zu reagieren.

Intensivstationen sind die fachlichen Anforderungen sehr hoch, so dass es häufig vorkommt, dass die externen Leiharbeitskräfte die an sie gestellten Anforderungen nicht erfüllen können. Dieses Wissen verursacht bei den Stammbeschäftigten zusätzlichen Stress, da während des Dienstes dann der Gedanke „hoffentlich geht alles gut“ ständig präsent ist (05PK06_2010). 


\section{Schlussfolgerungen und Ausblick}

Ausgangspunkt der Untersuchung ist ein aktueller Wandel in der Patientenversorgung, der sich an einer Neu-Definition dessen, welche Standards im öffentlichen Diskurs und in der Praxis dabei zugrunde gelegt werden, ablesen lässt. Während die fordistische Phase in erster Linie durch eine Orientierung geprägt war, die auf eine Demokratisierung der medizinischen Versorgung setzte und dabei den Mensch in den Mittelpunkt medizinisch-pflegerischer Entscheidungen und Handeln rückte, erfolgt die Ausrichtung des Gesundheitswesens seit Mitte der 1980er Jahre vor allen an marktzentrierten Steuerungsinstrumenten und -prinzipien, die zu einer zunehmenden Kommodifizierung von Gesundheitsdienstleistungen führen. Die Folgen dieses Umbruchs sind vor allem auch im Pflegebereich spürbar. Hier treffen bestehende berufsethische Orientierungen auf neue Marktzwänge mit der Konsequenz, dass sich für die PflegerInnen die Möglichkeiten, ihre Arbeit den tradierten Ansprüchen gemäß auszurichten, immer mehr verengen. Wie gezeigt wurde, sind die PflegerInnen mit einer halbierten Marktlogik konfrontiert: Zwar werden die neuen ,Imperative des Marktes“ ganz unmittelbar an sie weitergegeben, im Gegenzug werden ihnen jedoch nicht etwa auch Handlung erweiternde Freiheiten geboten. Ganz im Gegenteil unterliegt ihr Arbeitshandeln immer weitreichenderen Restriktionen. Zugleich wird von ihnen auch erwartet, dass sie die Versorgungsqualität trotz Rationalisierungsmaßnahmen und Umbauten der stationären und ambulanten Einrichtungen nach Kosten-NutzenKalkülen aufrechterhalten.

Vor dem Hintergrund dieser Befunde ist zu fragen, wie lange die aufgezeigten Widersprüche bei der Patientenversorgung weiterhin von den PflegerInnen abgepuffert werden und ob diese nicht über kurz oder lang beginnen, ihre sozialethischen Prinzipien der von ,Marktzwängen' geprägten Realität anzupassen. Betrachtet man die Reaktionsweisen dieser Beschäftigtengruppe systematisch, so zeigt sich u.a., dass einige Pflegekräfte aus Gründen des Selbstschutzes und einer drohenden Demoralisierung sich von ihrer ausgeprägten berufsethischen Orientierung distanzieren und stattdessen auf verschiedene Varianten des inneren Exits (innere Kündigung oder auch Wechsel des Arbeitsgebers) zurückgreifen.

\section{Literaturverzeichnis}

Badura, B. (1995): Gesundheitsdienstleistungen im Wandel, in: H.-J. Bullinger (Hrsg.): Dienstleistung der Zukunft. Märkte, Unternehmen und Infrastruktur im Wandel, Wiesbaden: Gabler, 183-190.

Badura, B. (1990): Interaktionsstreß. Zum Problem der Gefühlsregulierung in der modernen Gesellschaft, in: Zeitschrift für Soziologie, Jg. 19/Heft 5, 317-328.

Bär, S. (2011): Das Krankenhaus zwischen ökonomischer und medizinischer Vernunft, Wiesbaden, Heidelberg: VS Verlag für Sozialwissenschaften.

Becker, K./Brinkmann, U./Engel, T. (2012): Arbeits- und Gesundheitsschutz \& Leiharbeit - zwei problematische Handlungsfelder, in: Arbeit, Jg. 21/Heft 1, 20-38.

Becker, K./Engel, T. (Hrsg.) (2011): Ambulante Pflegearbeit unter Druck. Ergebnisse einer Beschäftigtenbefragung zu gesundheitsbelastenden und -förderlichen Arbeitsbedingungen 
in Thüringen (=Jenaer Beiträge zur Soziologie, 22), Jena: Universität Jena Institut für Soziologie.

Binswanger, M. (2010): Sinnlose Wettbewerbe. Warum wir immer mehr Unsinn produzieren, Freiburg: Herder.

Böhle, F. (1999): Nicht nur mehr Qualität, sondern auch höhere Effizienz - Subjektivierendes Arbeitshandeln in der Altenpflege, in: Zeitschrift für Arbeitswissenschaft, Jg. 53/Heft 3, 174-181.

Bräutigam, C./Dablbeck, E./Enste, P./Evans, M./Hilbert, J. (2010): Flexibilisierung und Leiharbeit in der Pflege, Arbeitspapier der Hans-Böckler-Stiftung, 215.

Brinkmann, U. (2011): Die unsichtbare Faust des Marktes. Betriebliche Kontrolle und Koordination im Finanzmarktkapitalismus, Berlin: Edition Sigma.

Brucks, U. (1999): Gefühlsarbeit - Versuch einer Begriffsklärung, in: Zeitschrift für Arbeitswissenschaft, Jg. 53/Heft 3, 182-186.

Büssing, A./Glaser, J. (1999): Work Stressors in Nursing in the Course of Redesign. Implications for Burnout and Interactional Stress, in: European Journal of Work and Organizational Psychology, Vol. 8/No. 3, 401-426.

Bundesärztekammer (2012): Beschlussprotokoll des 112. Deutschen Ärztetages in Mainz, 9.22.05.2009. Link: http://www.bundesaerztekammer.de/page.asp?his=0.2.23.7184 (zuletzt abgerufen am 28.06.2012).

Dunkel, W./Weibrich, M. (2010): Arbeit als Interaktion, in: Böhle, F./Voß, G. G./Wachtler, G. (Hrsg.): Handbuch Arbeitssoziologie, Wiesbaden: VS Verlag für Sozialwissenschaften, $177-200$.

Eckstein, W. (Hrsg.) (1926): Adam Smith: Theorie der ethischen Gefühle, Bd. 1, Leipzig: Felix Meiner.

Freidson, E./Rohde, J. J./Schoene, W. (1979): Der Ärztestand. Berufs- und wissenschaftssoziologische Durchleuchtung einer Profession, Stuttgart: Enke.

Gerlinger, T./Mosebach, K. (2009): Die Ökonomisierung des deutschen Gesundheitswesens. Ursachen, Ziele und Wirkungen wettbewerbsbasierter Kostendämpfungspolitik, in: Böhlke, N./Gerlinger, T./Mosebach, K./Schmucker, R./Schulten, T. (Hrsg.): Privatisierung von Krankenhäusern. Erfahrungen und Perspektiven aus Sicht der Beschäftigten, Hamburg: VSA-Verlag, 10-43.

Glaser, J. (2006): Arbeitsteilung, Pflegeorganisation und ganzheitliche Pflege - arbeitsorganisatorische Rahmenbedingungen für Interaktionsarbeit in der Pflege, in: Böhle, F. (Hrsg.): Arbeit in der Interaktion - Interaktion als Arbeit. Arbeitsorganisation und Interaktionsarbeit in der Dienstleistung, Wiesbaden: VS Verlag für Sozialwissenschaften, 43-59.

Hacker, W. (2005): Arbeitspsychologie, Bern: Hans Huber.

Hirsch, J./Roth, R. (1986): Das neue Gesicht des Kapitalismus. Vom Fordismus zum PostFordismus, Hamburg: VSA-Verlag.

Hochschild, A. R. (2003): The Managed Heart. Commercialization of Human Feeling, Twentieth Anniversary Edition, Berkeley, Los Angeles, London: California Press.

Irrgang, B. (1995): Grundriß der medizinischen Ethik, München: UBT Reinhardt.

Jorzig, A./Uphoff, R. (2010): Delegation und Substitution - wenn der Pfleger den Doktor ersetzt, Berlin, Heidelberg: Springer.

Juchli, L. (1994): Pflege. Praxis und Theorie der Gesundheits- und Krankenpflege, 7. Auflage, Stuttgart: Georg Thieme Verlag.

Kliemt, H. (2006): Budgetierung, Standardisierung, Priorisierung, in: Schöne-Seifert, B. (Hrsg.): Gerecht behandelt? Rationierung und Priorisierung im Gesundheitswesen, Paderborn: Mentis, 91-107. 
Kühn, H. (2004): Die Ökonomisierungstendenz in der medizinischen Versorgung, in: Elsner, G./Gerlinger, T./Stegmüller, K. (Hrsg.): Markt versus Solidarität. Gesundheitspolitik im deregulierten Kapitalismus, Hamburg: VSA-Verlag, 25-42.

Müller-Jentsch, W. (1993): Konfliktpartnerschaft. Akteure und Institutionen der industriellen Beziehungen, München: Hampp.

Nerdinger, F. W./Röper, M. (1999): Emotionale Dissonanz und Burnout. Eine empirische Untersuchung im Pflegebereich eines Universitätskrankenhauses, in: Zeitschrift für Arbeitswissenschaft, Jg. 53/Heft 53, 187-193.

o.V. (2012): Ärzte lehnen geplante Klinikempfehlung durch Kassen strikt ab. Link: https://www.aerzteblatt.de/nachrichten/50394/Aerzte-lehnen-geplante-

Klinikempfehlung-durch-Kassen-strikt-ab (zuletzt abgerufen am 03.09.2012).

Rieder, K. (1999): Zwischen Lohnarbeit und Liebesdienst. Belastungen in der Krankenpflege, Weinheim, München: Juventa Verlag.

Sachverständigenrat zur Begutachtung der Entwicklung im Gesundheitswesen (1991): Gutachten 1991, Baden-Baden: Nomos Verlagsgesellschaft.

Sachverständigenrat zur Begutachtung der Entwicklung im Gesundheitswesen (2007): Gutachten 2007.

Link: https:www.svr-gesundheit.de/index.php?id=15 (zuletzt abgerufen am 31.10.2012).

Sachverständigenrat zur Begutachtung der Entwicklung im Gesundheitswesen (2012): Sondergutachten 2012. Link: https:www.svr-gesundheit.de/index.php?id=378 (zuletzt abgerufen am 31.10.2012).

Schöne-Seifert, B. (2006): Gerecht behandelt? Rationierung und Priorisierung im Gesundheitswesen, Paderborn: Mentis.

Schwartz, F. W./Angerer, P. (2010): Arbeitsbedingungen und Befinden von Ärztinnen und Ärzten. Befunde und Interventionen, Köln: Deutscher Ärzte-Verlag.

Simon, M. (2008): Sechzehn Jahre Deckelung der Krankenhausbudgets. Eine kritische Bestandsaufnahme. Studie im Auftrag von ver.di. Hannover.

Smith, A. (1782/83): Wealth of Nations, London: W. Strahan und T. Cadell.

Strauss, A./Fagerhaugh, S./Suczek, B./Wiener, C. (1982): Sentimental Work in the Technologized Hospital, in: Sociology of Health and Illness, Vol. 4/No. 3, 254-278.

Toffler, A. E. (1980): The Third Wave, New York: W. Morrow.

Vogd, W. (2006): Die Organisation Krankenhaus im Wandel. Eine dokumentarische Evaluation aus Sicht der ärztlichen Akteure, Bern: Hans Huber.

Voß, G./Rieder, K. (2005): Der arbeitende Kunde. Wenn Kunden zu unbezahlten Mitarbeitern werden, Frankfurt a.M., New York: Campus.

White M. D. (2010): Markets and Dignity. The Essential Link (with an Application to Health Care); in: White, M: (Ed.): Accepting the Invisible Hand. Market-Based Approaches to Social-Economic Problems, Basingstoke: Palgrave Macmillan, 1-23.

Zapf, D. (2002): Emotion Work and Psychological Well-Being. A Review of the Literature and Some Conceptual Considerations, in: Human Resource Management Review, Vol. 12/No. 2, 237-268. 